\title{
Displacement Operator Formalism
}

\section{Joannis Papavassiliou*}

Departamento de Física Teòrica and IFIC Centro Mixto, Universidad de Valencia-CSIC, E-46100, Burjassot, Valencia, Spain

E-mail: Joannis.Papavassiliouduv.es

\section{Daniele Binosi}

ECT*, Villa Tambosi, Strada delle Tabarelle 286 I-38050 Villazzano (Trento), Italy

I.N.F.N., Gruppo Collegato di Trento, Trento, Italy

E-mail: binosi@ect.it

\section{Apostolos Pilaftsis}

CERN, Physics Department, Theory Division, CH-1211 Geneva 23, Switzerland, School of Physics and Astronomy, University of Manchester,

Manchester M13 9PL, United Kingdom

E-mail: Apostolos.Pilaftsis@manchester.ac.uk

We review the $D$-formalism, a new method for determining the renormalization of Green functions to all orders in perturbation theory. This formalism exploits the fact that the renormalized Green functions may be calculated by displacing by an infinite amount the renormalized fields and parameters of the theory with respect to the unrenormalized ones. We briefly discuss some of its main characteristics, its conceptual and practical advantages, and some new results obtained through it.

International Europhysics Conference on High Energy Physics

July 21st - 27th 2005

Lisboa, Portugal

\footnotetext{
${ }^{*}$ Speaker.
} 
We review a new formalism, the Displacement Operator Formalism, or the $D$-formalism, which enables one to systematically organize and explicitly compute the counterterms (CT) involved in the renormalization procedure, to all orders in perturbation theory. The central observation, leading to this new formulation, is that the effect of renormalizing any given Green function may be expressed in terms of ultraviolet infinite displacements caused by the renormalization on both the fields and the parameters of the theory. Specifically, these UV infinite displacements, or shifts, quantify the difference between fields and parameters before and after renormalization. We will present the derivation of the D-formalism in the context of a $\lambda \phi^{4}$ theory; for the generalization to more involved field theories, see [1].

Let $\Gamma_{\phi^{n}}$ be a bare, one-particle irreducible $n$-point Green function. After carrying out the renormalization programme, in $d=4-\varepsilon$ dimensions, one will have that

$$
\phi^{n} \Gamma_{\phi^{n}}\left(\lambda, m^{2} ; \mu, \varepsilon\right)=\phi_{\mathrm{R}}^{n} \Gamma_{\phi^{n}}^{\mathrm{R}}\left(\lambda_{\mathrm{R}}, m_{\mathrm{R}}^{2} ; \mu\right),
$$

where $\lambda, m^{2}, \phi$ (respectively $\lambda_{\mathrm{R}}, m_{\mathrm{R}}^{2}, \phi_{\mathrm{R}}$ ) are the bare (respectively renormalized) parameters and dynamical field of the theory at hand,

$$
\phi=Z_{\phi}^{\frac{1}{2}} \phi_{\mathrm{R}}, \quad \lambda=Z_{\lambda} \lambda_{\mathrm{R}}, \quad m^{2}=Z_{m^{2}} m_{\mathrm{R}}^{2},
$$

and $\Gamma_{\phi^{n}}^{\mathrm{R}}\left(\lambda_{\mathrm{R}} ; \mu\right)$ represents the renormalized $n$-legs Green function. Then, we may rewrite the lefthand side of (1) as

$$
\phi^{n} \Gamma_{\phi^{n}}\left(\lambda, m^{2} ; \mu, \varepsilon\right)=\left(\phi_{\mathrm{R}}+\delta \phi\right)^{n} \Gamma_{\phi^{n}}\left(\lambda_{\mathrm{R}}+\delta \lambda, m_{\mathrm{R}}^{2}+\delta m^{2} ; \mu, \varepsilon\right),
$$

where the parameter shifts

$$
\delta \phi=\left(Z_{\phi}^{\frac{1}{2}}-1\right) \phi_{\mathrm{R}}, \quad \delta \lambda=\left(Z_{\lambda}-1\right) \lambda_{\mathrm{R}}, \quad \delta m^{2}=\left(Z_{m^{2}}-1\right) m_{\mathrm{R}}^{2}
$$

quantify the difference of the renormalized quantities with respect to the corresponding unrenormalized ones in a given renormalization scheme R. Notice that our formulation treats fields and couplings on equal footing, i.e., as independent fundamental parameters of the theory.

Next we use a Taylor expansion to trade the combinations $\phi_{\mathrm{R}}+\delta \phi, \lambda_{\mathrm{R}}+\delta \lambda$, and $m_{\mathrm{R}}+\delta m$, for the renormalized parameters $\phi_{\mathrm{R}}, \lambda_{\mathrm{R}}$, and $m_{\mathrm{R}}$. To this end, it is natural to introduce the differential displacement operator

$$
D=\delta \phi \frac{\partial}{\partial \phi_{\mathrm{R}}}+\delta \lambda \frac{\partial}{\partial \lambda_{\mathrm{R}}}+\delta m^{2} \frac{\partial}{\partial m_{\mathrm{R}}^{2}},
$$

in which the shifts are treated as independent parameters, and only at the very end of all the manipulations they will be replaced by their actual values in terms of the renormalization constants $Z_{\phi}\left(\lambda_{\mathrm{R}}, m_{\mathrm{R}}^{2} ; \varepsilon\right), Z_{\lambda}\left(\lambda_{\mathrm{R}}, m_{\mathrm{R}}^{2} ; \varepsilon\right)$, and $Z_{m}\left(\lambda_{\mathrm{R}}, m_{\mathrm{R}}^{2} ; \varepsilon\right)$. It is then not difficult to derive the following (all-order) master equation

$$
\left(\phi_{\mathrm{R}}+\delta \phi\right)^{n} \Gamma_{\phi^{n}}\left(\lambda_{\mathrm{R}}+\delta \lambda, m_{\mathrm{R}}^{2}+\delta m^{2} ; \mu, \varepsilon\right)=\left\langle e^{D} \phi_{\mathrm{R}}^{n} \Gamma_{\phi^{n}}\left(\lambda_{\mathrm{R}}, m_{\mathrm{R}}^{2} ; \mu, \varepsilon\right)\right\rangle,
$$

or equivalently

$$
\phi_{\mathrm{R}}^{n} \Gamma_{\phi^{n}}^{\mathrm{R}}\left(\lambda_{\mathrm{R}}, m_{\mathrm{R}}^{2} ; \mu\right)=\left\langle e^{D} \phi_{\mathrm{R}}^{n} \Gamma_{\phi^{n}}\left(\lambda_{\mathrm{R}}, m_{\mathrm{R}}^{2} ; \mu, \varepsilon\right)\right\rangle,
$$


where $\langle\ldots\rangle$ means that the CT parameters $\delta \phi, \delta \lambda$, and $\delta m^{2}$ are to be set to their actual values according to (4) after the action of the $D$ operator. It is important to appreciate at this point the inherent non-perturbative nature of the above formulation, manifesting itself through the exponentiation of the $D$ operator. Perturbative results (at arbitrary order) may be recovered as a special case through an appropriate order-by-order expansion of the above master formula, In doing so, one should notice that not only $\Gamma_{\phi^{n}}$ should be expanded starting from tree level, but, accordingly, also the shifts $\delta \phi, \delta \lambda$ and $\delta m^{2}$, together with the displacement operator $D$

Since the shifts are treated as independent parameters, the displacement operator at different perturbative orders commute, and one can use the ordinary Taylor expansion for the exponentiation of $D$; up to three loops, one then gets

$$
e^{D}=1+D^{(1)}+\left(D^{(2)}+\frac{1}{2} D^{(1) 2}\right)+\left(D^{(3)}+D^{(2)} D^{(1)}+\frac{1}{6} D^{(1) 3}\right)+\ldots,
$$

with

$$
D^{(n)}=\delta \phi^{(n)} \frac{\partial}{\partial \phi_{\mathrm{R}}}+\delta \lambda^{(n)} \frac{\partial}{\partial \lambda_{\mathrm{R}}}+\delta m^{2(n)} \frac{\partial}{\partial m_{\mathrm{R}}^{2}} .
$$

The parameter shifts $\delta \phi^{(n)}, \delta \lambda^{(n)}$, and $\delta m^{2(n)}$ are loop-wise defined as follows:

$$
\delta \phi^{(n)}=Z_{\phi}^{\frac{1}{2}(n)} \phi_{\mathrm{R}}, \quad \delta \lambda^{(n)}=Z_{\lambda}^{(n)} \lambda_{\mathrm{R}}, \quad \delta m^{2(n)}=Z_{m^{2}}^{(n)} m_{\mathrm{R}}^{2} .
$$

By acting with the operator $e^{D}$ on the 1PI correlation functions $\phi_{\mathrm{R}}^{n} \Gamma_{\phi^{n}}\left(\lambda_{\mathrm{R}}, m_{\mathrm{R}}^{2} ; \mu, \varepsilon\right)$, we can easily determine the expressions for the renormalized correlation functions $\phi_{\mathrm{R}}^{n} \Gamma_{\phi^{n}}^{\mathrm{R}}\left(\lambda_{\mathrm{R}}, m_{\mathrm{R}}^{2} ; \mu\right)$ at the one-, and two-loop level, which read

$$
\begin{aligned}
\phi_{\mathrm{R}}^{n} \Gamma_{\phi^{n}}^{\mathrm{R}(1)}\left(\lambda_{\mathrm{R}}, m_{\mathrm{R}}^{2} ; \mu\right)= & \left\langle D^{(1)} \phi_{\mathrm{R}}^{n} \Gamma_{\phi^{n}}^{(0)}\left(\lambda_{\mathrm{R}}, m_{\mathrm{R}}^{2} ; \mu\right)\right\rangle+\phi_{\mathrm{R}}^{n} \Gamma_{\phi^{n}}^{(1)}\left(\lambda_{\mathrm{R}}, m_{\mathrm{R}}^{2} ; \mu, \varepsilon\right), \\
\phi_{\mathrm{R}}^{n} \Gamma_{\phi^{n}}^{\mathrm{R}(2)}\left(\lambda_{\mathrm{R}}, m_{\mathrm{R}}^{2} ; \mu\right)= & \left\langle\left(D^{(2)}+\frac{1}{2} D^{(1) 2}\right) \phi_{\mathrm{R}}^{n} \Gamma_{\phi^{n}}^{(0)}\left(\lambda_{\mathrm{R}}, m_{\mathrm{R}}^{2} ; \mu\right)+D^{(1)} \phi_{\mathrm{R}}^{n} \Gamma_{\phi^{n}}^{(1)}\left(\lambda_{\mathrm{R}}, m_{\mathrm{R}}^{2} ; \mu, \varepsilon\right)\right\rangle \\
& +\phi_{\mathrm{R}}^{n} \Gamma_{\phi^{n}}^{(2)}\left(\lambda_{\mathrm{R}}, m_{\mathrm{R}}^{2} ; \mu, \varepsilon\right) .
\end{aligned}
$$

The above identities furnish the exact expressions for the various renormalization constants, to any given order. Specifically, the renormalization constants appearing in the $D$-operator [viz.(5)] are determined through a system of algebraic equation; the latter is obtained by considering all divergent Green's function $\Gamma_{\phi^{n}}$ of the theory, and demanding that the right-hand side of (11) be free of cutoff-dependent terms (i.e. no $1 / \varepsilon$ terms in dimensional regularization).

Let us now list some of the main characteristics and advantages of the $D$-formalism, together with a new, highly non-trivial result obtained through it, and some possible future applications.

(i) The $D$-formalism allows one to to determine unambiguously the CTs to any given order in perturbation theory: they are automatically obtained through the straightforward application of the $D$ operator on the unrenormalized Green functions, without having to resort to any additional arguments whatsoever. The usual diagrammatic representation of the CTs is reproduced exactly, through the action of the $D$ operator on the Feynman graphs determining the given Green function before the integration over the virtual loop momenta is carried out. This is clearly an advantage, at least from the logistical point of view, because it reduces significantly the number of Feynman 
graphs that need be considered at each order. If one instead acts with $D$ after the momentum integration has been performed one loses this direct diagrammatic interpretation, but recovers the same final answer for the renormalized Green function.

(ii) The above features are particularly relevant in the context of gauge theories, or special gauge fixing schemes (such as the background field method [3]) where keeping track of the CTs related to the gauge-fixing parameter $(\xi)$ may be conceptually subtle. All such CT are accounted for unambiguously by introducing into the $D$ operator a term of the form $\delta \xi \frac{\partial}{\partial \xi_{R}}$, and subsequently acting on the corresponding Green's function.

(iii) When using (11) note that one must only include Feynman diagrams without counterterms, i.e. only those contributing to the bare Green's functions. The corresponding diagrams containing CT will be generated dynamically by the subsequent action of the $D$ operator. The fact that the procedure generates precisely all necessary $\mathrm{CT}$ is reflected in the fact that the resulting final expression are free of overlapping divergences.

(iv) The control that the $D$-formalism provides on the structure and organization of the CTs to all orders in perturbation, has allowed us to obtain the exact form of the deformations induced due to the renormalization procedure to any type of relations or constraints which are valid at the level of unrenormalized Green functions. In particular, as was shown in detail in [1], the straightforward application of the $D$-formalism yields, for the first time, the deformation of the Nielsen Identities [2] in a closed, and, in principle, calculable form. This new result furnishes the exact dependence of the renormalized Green functions on the renormalized gauge-fixing parameter to all orders.

(v) This formulation opens novel perspectives for the study of several other known topics. Specifically, the $D$-formalism may be used to systematically investigate the renormalization-scheme dependence of correlation functions. It may also be employed to algebraically determine the restoring terms of a "bad" UV regularizing scheme, i.e., a scheme that does not preserve the SlavnovTaylor identities [4]. Since it provides all-order information on the renormalization of Green functions under study, it might be useful in controlling the calculation of non-perturbative effects, such as those related to the dynamics of renormalons. The $D$-formalism can be straightforwardly extended to systematize the procedure of renormalizing non-renormalizable field theories. In particular, it may be used to organize the infinite series of CTs needed to renormalize such theories. Even in the case of renormalizable perturbative field theories the $D$-formalism can be automated, for example with the aid of a computational package, to reliably compute all the CTs required for the renormalization of 1PI correlation functions at high orders.

\section{Acknowledgments}

This work was supported by the MCyT grant FPA2002-00612

\section{References}

[1] D. Binosi, J. Papavassiliou and A. Pilaftsis, Phys. Rev. D 71, 085007 (2005) [arXiv:hep-ph/0501259].

[2] N. K. Nielsen, Nucl. Phys. B 97, 527 (1975).

[3] L. F. Abbott, Nucl. Phys. B 185, 189 (1981).

[4] A. A. Slavnov, Theor. Math. Phys. 10, 99 (1972) [Teor. Mat. Fiz. 10, 153 (1972)]; J. C. Taylor, Nucl. Phys. B 33, 436 (1971). 